\title{
Neutropenia as a prognostic factor and safety of second-line therapy with S-1 for advanced or recurrent pancreatic cancer
}

\author{
MAKIKO IKAGAWA $^{1}$, MICHIO KIMURA ${ }^{2}$, MINA IWAI $^{2}$, EISEKI USAMI $^{2}$, \\ TOMOAKI YOSHIMURA $^{2}$ and KIMIO YASUDA ${ }^{1}$ \\ ${ }^{1}$ Faculty of Pharmaceutical Sciences, Kinjo Gakuin University, Nagoya, Aichi 463-8521; \\ ${ }^{2}$ Department of Pharmacy, Ogaki Municipal Hospital, Ogaki, Gifu 503-8502, Japan
}

Received June 5, 2015; Accepted June 17, 2016

DOI: $10.3892 / \mathrm{mco} .2016 .940$

\begin{abstract}
The aim of this retrospective study was to investigate the safety of S-1 as second-line therapy and to evaluate the association between neutropenia occurring during first-line gemcitabine (GEM) therapy and survival for advanced or recurrent pancreatic cancer (APC). Between January, 2010 and December, 2014, 123 APC patients received chemotherapy at the Ogaki Municipal Hospital (Ogaki, Japan). Of those, 37 received GEM as first-line and S-1 as a second-line therapy (GEM $\rightarrow \mathrm{S}-1$ group). A further 60 patients received GEM as first-line therapy, but did not receive second-line therapy (GEM group). The median overall survival in the $\mathrm{GEM} \rightarrow \mathrm{S}-1 \quad(\mathrm{n}=37)$ and GEM $(\mathrm{n}=60)$ groups was 323 days [95\% confidence interval (CI): 138-218.9 days] and 172 days (95\% CI: $105-184.4$ days), respectively $(\mathrm{P}=0.0004)$. The median overall survival in the mild (grade $\leq 2 ; n=63$ ) and severe ( grade $\geq 3$; $n=34$ ) neutropenia groups was 178 days (95\% CI: 182-275 days) and 330 days (95\% CI: 297-514 days), respectively (log-rank test, $\mathrm{P}=0.0023$ ). The severe non-haematological toxicities associated with $\mathrm{S}-1$ as second-line therapy were nausea $(2.7 \%)$ and hand-foot syndrome $(2.7 \%)$. Second-line S-1 treatment was discontinued due to adverse events in $5.4 \%(2 / 37)$ of the cases. In conclusion, neutropenia occurring during GEM therapy administered as first-line treatment to APC patients was strongly associated with a better prognosis. S-1 therapy as second-line treatment was associated with a low incidence of severe adverse events and the patients were able to successfully continue treatment.
\end{abstract}

Correspondence to: Dr Michio Kimura, Department of Pharmacy, Ogaki Municipal Hospital, 4-86 Minaminokawa-Cho, Ogaki, Gifu 503-8502, Japan

E-mail: kimkim0305nao@yahoo.co.jp

Key words: gemcitabine, S-1, adherence, neutropenia, pancreatic cancer

\section{Introduction}

Pancreatic cancer has the worst prognosis among all refractory gastrointestinal cancers. According to data on the number of site-specific cancer deaths in Japan, pancreatic is the fourth most common cancer, after lung, stomach and colon cancers (1). In 1997, randomised clinical trials comparing gemcitabine (GEM) and 5-fluorouracil (5-FU) chemotherapy for pancreatic cancer (2), demonstrated that GEM was more beneficial for symptom relief compared with 5-FU, and also prolonged survival. In addition, since August, 2006, an oral 5-FU formulation containing tegafur, gimeracil and oteracil potassium (S-1) has been approved by insurance companies for the treatment of pancreatic cancer. The GEM and S-1 trial (GEST study) demonstrated that S-1 was non-inferior to GEM, but did not prove the superiority of combination therapy with GEM and S-1 (3). Therefore, GEM or S-1 is recommended for standard chemotherapy of advanced or recurrent pancreatic cancer (APC).

The incidence of myelosuppression, such as neutropenia, in first-line GEM therapy is high, which may delay treatment and affect prognosis. Moreover, neutrophil count $(4,5)$ and the ratio of neutrophils to lymphocytes $(6,7)$ have been reported to be prognostic factors for APC patients. In addition, it has been reported that neutropenia is a prognostic factor in gastric (8) and colon cancers (9), as well as haematopoietic tumours (10). However, due to the poor prognosis of pancreatic cancer, the association between neutropenia and prognosis, and details such as dose and relative dose intensity (RDI), have not been investigated in the clinical setting. Furthermore, in the GEST study (3), gastrointestinal symptoms such as nausea, diarrhoea and stomatitis have been frequently observed among adverse events (AEs) associated with S-1 monotherapy. Thus, when administering S-1 as second-line therapy, tolerability to AEs may be reduced, with deterioration of the patient's condition. The frequency of AEs and treatment continuity associated with second-line S-1 chemotherapy have not been extensively investigated (11-14). We previously reported that albumin (Alb) levels $<3.5 \mathrm{~g} / \mathrm{dl}$ and creatinine clearance levels $<78 \mathrm{ml} / \mathrm{min}$ were risk factors for treatment discontinuation or dosage reduction of $\mathrm{S}-1$ in gastric cancer chemotherapy $(15,16)$.

Therefore, this retrospective study aimed to investigate the safety of S-1 as second-line therapy for APC patients. In 
addition, we evaluated the association between neutropenia occurring during first-line GEM therapy and survival.

\section{Subjects and methods}

Subjects and methods. Between January, 2010 and December, 2014, 123 patients received chemotherapy for APC at the Ogaki Municipal Hospital (Ogaki, Japan). Of those, 37 received GEM as first-line and S-1 as second-line therapy (GEM $\rightarrow \mathrm{S}-1$ group). A further 60 patients received GEM as first-line therapy, but did not receive second-line therapy (GEM group). Age, RDI, administration period, AEs and reasons for dose reduction or temporary suspension of medication were retrospectively surveyed for each patient. In addition, patients receiving ongoing treatment with GEM or S-1 during the study period were excluded. The dates of AEs and reasons for discontinuation of chemotherapy were extracted from electronic charts. The severity of AEs was classified according to the Common Terminology Criteria for Adverse Events, version 4.0 (http://evs.nci.nih.gov/ftp1/ CTCAE/CTCAE_4.03_2010-06-14_QuickReference_5x7. pdf). The present study was approved by the Institutional Review Board of Ogaki Municipal Hospital.

Doses and routes of GEM and S-1 therapies. GEM was administered intravenously at a starting dose of $1,000 \mathrm{mg} / \mathrm{m}^{2}$ over $30 \mathrm{~min}$, weekly, on days 1,8 and 15 over a 4 -week period. S-1 was orally administered for 4 weeks (dose: $<1.25$ $\mathrm{m}^{2}$ of body surface area, $80 \mathrm{mg} / \mathrm{d} ; 1.25-1.5 \mathrm{~m}^{2}, 100 \mathrm{mg} / \mathrm{d}$; $\geq 1.5 \mathrm{~m}^{2}, 120 \mathrm{mg} / \mathrm{d}$ ), followed by a 2 -week washout period.

Statistical analysis. The F-test was performed to compare the two groups. Welch's t-test or the Chi-square test of independence (Fisher's exact probability test) was used to analyse the patients' characteristics (age, neutrophil count, RDI and dosage) shown in Table I. The Kaplan-Meier log-rank test was used to compare overall survival. In all these tests, $\mathrm{P}<0.05$ was considered to indicate statistically significant differences. All statistical analyses were performed using JMP 8 software (SAS Institute Inc., Cary, NC, USA).

\section{Results}

Patient characteristics. The patients' characteristics are shown in Table I. In the GEM $\rightarrow \mathrm{S}-1$ and GEM groups, the median age was 68 years (range, 54-77 years) and 66.3 years (range, 43-83 years); the median neutrophil count was 3,650/ $\mu 1$

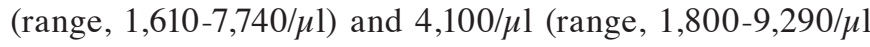
$(\mathrm{P}=0.0431)$; the RDI was $90.4 \%$ (range, $36.9-100 \%$ ) and $83.4 \%$ (range, $53.0-100 \%$ ); and the dosage used was $100 \%$ (range, $74.6-100 \%$ ) and $100 \%$ (range, $77.1-100 \%$ ), respectively.

Overall survival in the $G E M \rightarrow S-1$ and $S-1$ groups. The Kaplan-Meier survival curves for the cohorts $(\mathrm{n}=97)$ are shown in Fig. 1. The median overall survival of the $\mathrm{GEM} \rightarrow \mathrm{S}-1 \quad(\mathrm{n}=37)$ and GEM $(\mathrm{n}=60)$ groups were 323 days [95\% confidence interval (CI): 138-218 days] and 172 days (95\% CI: 105-184 days), respectively (log-rank test, $\mathrm{P}=0.0004)$.
Overall survival according to the highest grade of neutropenia following first-line therapy with GEM. The Kaplan-Meier survival curves showing the highest grade of neutropenia following first-line therapy with GEM ( $n=97)$ are shown in Fig. 2. The median overall survival time in the mild (grade $\leq 2 ; n=63$ ) and severe (grade $\geq 3 ; n=34$ ) neutropenia groups was 178 days (95\% CI: $182-275$ days) and 330 days (95\% CI: 297-514 days), respectively (log-rank test, $\mathrm{P}=0.0023)$. In addition, the frequency of grade 3 or 4 neutropenia in the GEM $\rightarrow \mathrm{S}-1$ group $(48.6 \%, 18 / 37$ cases) was significantly higher compared with that in the GEM group $(26.7 \%, 16 / 60$ cases; $\mathrm{P}=0.0238)$.

Reasons for discontinuation, postponement and dose reduction. The reasons for discontinuation, postponement and dose reduction in the GEM $\rightarrow \mathrm{S}-1$ and GEM groups are shown in Table II. In the GEM $\rightarrow \mathrm{S}-1$ group, GEM administration was interrupted due to progressive disease (PD) in 36 cases, or AEs in 1 case. S-1 discontinuation occurred due to changes in performance status (PS), PD, AEs (diarrhoea and anorexia), and other reasons in 21, 13, 2 and 1 cases, respectively. In addition, GEM administration was postponed due to haematological and non-haematological toxicities in 22 and 4 cases, respectively, and other reasons in 1 case. S-1 administration was postponed due to haematological toxicities in 3 cases; non-haematological toxicities in 6 cases (diarrhoea, stomatitis, skin hyperpigmentation, constipation, anorexia and vomiting); a decrease in PS in 2 cases; due to the patient's wishes in 1 case; and other reasons in 3 cases.

In the GEM group, the dosage was reduced due to a decrease in PS, myelosuppression, renal failure and other reasons in $8,1,1$ and 1 cases, respectively.

Main adverse events of second-line therapy with $S-1$. The main AEs caused by second-line therapy with $\mathrm{S}-1$ are shown in Table III. The most common haematological toxicities were oligochromemia (14 cases, 37.8\%), leukopenia (7 cases, $18.9 \%$ ) and neutropenia (6 cases, 16.2\%). Non-haematological toxicities included anorexia ( 7 cases, $18.9 \%$ ), diarrhoea ( 7 cases, $18.9 \%$ ), malaise (6 cases, 16.2\%), stomatitis (6 cases, $16.2 \%$ ), nausea ( 5 cases, $13.5 \%)$, watery eyes ( 5 cases, $13.5 \%$ ) and skin hyperpigmentation (4 cases, 10.8\%).

Association between the incidence of gastrointestinal toxicity and serum Alb in second-line therapy with $S-1$. Following second-line S-1 therapy in the GEM $\rightarrow \mathrm{S}-1$ group, the frequency of grade 2, 3 or 4 malaise and digestive system disorders in subjects with Alb $<3.5 \mathrm{~g} / \mathrm{dl}$ (10/14 cases) were significantly higher compared with those with Alb $\geq 3.5 \mathrm{~g} / \mathrm{dl}$ ( $2 / 23$ cases; $\mathrm{P}=0.0002)$. In patients where treatment was interrupted due to diarrhoea and nausea ( 2 cases), the Alb levels were 3.3 and $3.2 \mathrm{~g} / \mathrm{dl}$, respectively. In addition, S-1 therapy was postponed in 6 cases due to AEs such as diarrhoea, stomatitis, skin hyperpigmentation, anorexia and nausea. In 5 of 6 of these cases, the Alb level was $\leq 3.5 \mathrm{~g} / \mathrm{dl}$.

\section{Discussion}

The aim of this retrospective study was to investigate the safety of S-1 as second-line therapy, and to evaluate the association 
Table I. Patient characteristics.

\begin{tabular}{|c|c|c|c|}
\hline & $\begin{array}{l}\text { Patients who received } \\
\text { second-line treatment }\end{array}$ & $\begin{array}{l}\text { Patients who did not receive } \\
\text { second-line treatment }\end{array}$ & \\
\hline Characteristics & $\begin{array}{l}\mathrm{GEM} \rightarrow \mathrm{S}-1 \\
\quad(\mathrm{n}=37)\end{array}$ & $\begin{array}{l}\text { GEM } \\
(n=60)\end{array}$ & P-value \\
\hline Age, years (range) & $68(54-77)$ & $66.3(43-83)$ & 0.3738 \\
\hline Gender, $\mathrm{n}$ & & & 0.4681 \\
\hline Female & 18 & 31 & \\
\hline Male & 19 & 29 & \\
\hline $\mathrm{BSA}, \mathrm{m}^{2}$ & $1.45(1.12-1.93)$ & $1.48(1.22-1.81)$ & 0.5942 \\
\hline $\mathrm{CrCl}, \mathrm{ml} / \mathrm{min}$ & 74.5 (43.2-120.9) & $76.1(21.7-150.4)$ & 0.9632 \\
\hline Disease stage, $\mathrm{n}$ & & & 0.0845 \\
\hline IVa & 19 & 21 & \\
\hline $\mathrm{IVb}$ & 18 & 39 & \\
\hline Disease status, $\mathrm{n}$ & & & 0.0090 \\
\hline Unresectable & 22 & 50 & \\
\hline Recurrent & 15 & 10 & \\
\hline Neutrophils, $/ \mu 1$ & $3,650(1,610-7,740)$ & $4,100(1,800-9,290)$ & 0.0431 \\
\hline RDI of GEM (range) & $90.4(36.9-100)$ & $83.4(53.0-100)$ & 0.1162 \\
\hline $\begin{array}{l}\text { Administration period of GEM, } \\
\text { days (range) }\end{array}$ & $159(48-574)$ & $95(7-882)$ & 0.2759 \\
\hline Dosage of GEM, \% & $100(74.6-100)$ & $100(77.1-100)$ & 0.9264 \\
\hline Metastatic site, $\mathrm{n}$ & & & 0.2308 \\
\hline Liver & 12 & 25 & \\
\hline Lung & 4 & 3 & \\
\hline Peritoneum & 2 & 10 & \\
\hline Lymph nodes & 3 & 8 & \\
\hline Other & 1 & 9 & \\
\hline Complications, $\mathrm{n}$ & & & 0.2959 \\
\hline Hypertension & 17 & 16 & \\
\hline Hyperlipidaemia & 6 & 3 & \\
\hline Diabetes & 13 & 16 & \\
\hline Asthma & 5 & 1 & \\
\hline
\end{tabular}

GEM, gemcitabine; S-1, tegafur, gimeracil, and oteracil potassium; BSA, body surface area; $\mathrm{CrCl}$, creatinine clearance; RDI, relative dose intensity; GEM $\rightarrow \mathrm{S}-1$, group, patients who received GEM as first-line therapy and S-1 as second-line therapy; GEM group, patients who received GEM as first-line therapy and did receive second-line therapy.

between neutropenia occurring during first-line GEM therapy and survival in APC patients.

It has been reported that second-line treatment with S-1 monotherapy is associated with a better prognosis in APC patients $(4,12-14)$. Similarly, this study has demonstrated that it is important to use GEM and S-1 for the treatment of APC. Furthermore, a study by Shitara et al (8) reported that neutropenia occurring during weekly paclitaxel treatment administered as second-line therapy to advanced gastric cancer patients is strongly associated with a better prognosis. In this study, the prognosis of APC patients with grade $\geq 3$ neutropenia during first-line GEM therapy was good.

Regarding the association between neutropenia and prognosis, Shitara et al (8) hypothesized that neutropenia, an indicator of bone marrow suppression caused by a specific dose of a chemotherapeutic agent, may also be a surrogate marker indicating that the same dose is adequate for exerting an antitumor effect. If neutropenia is not present, it is possible that the patient has been administered too low a dose. In our study, no significant differences were found between the $\mathrm{GEM} \rightarrow \mathrm{S}-1$ and GEM groups with regard to RDI and dose, or when the dosages were reduced. However, the neutrophil count was high in the GEM group at the start of treatment. Fridlender et al (17) reported that neutrophils are involved in vascularisation and are associated with cancer metastasis and angiogenesis. Hatori et al (4) reported that the number of neutrophils present prior to the first GEM treatment is a prognostic factor. Additionally, it has been reported that 


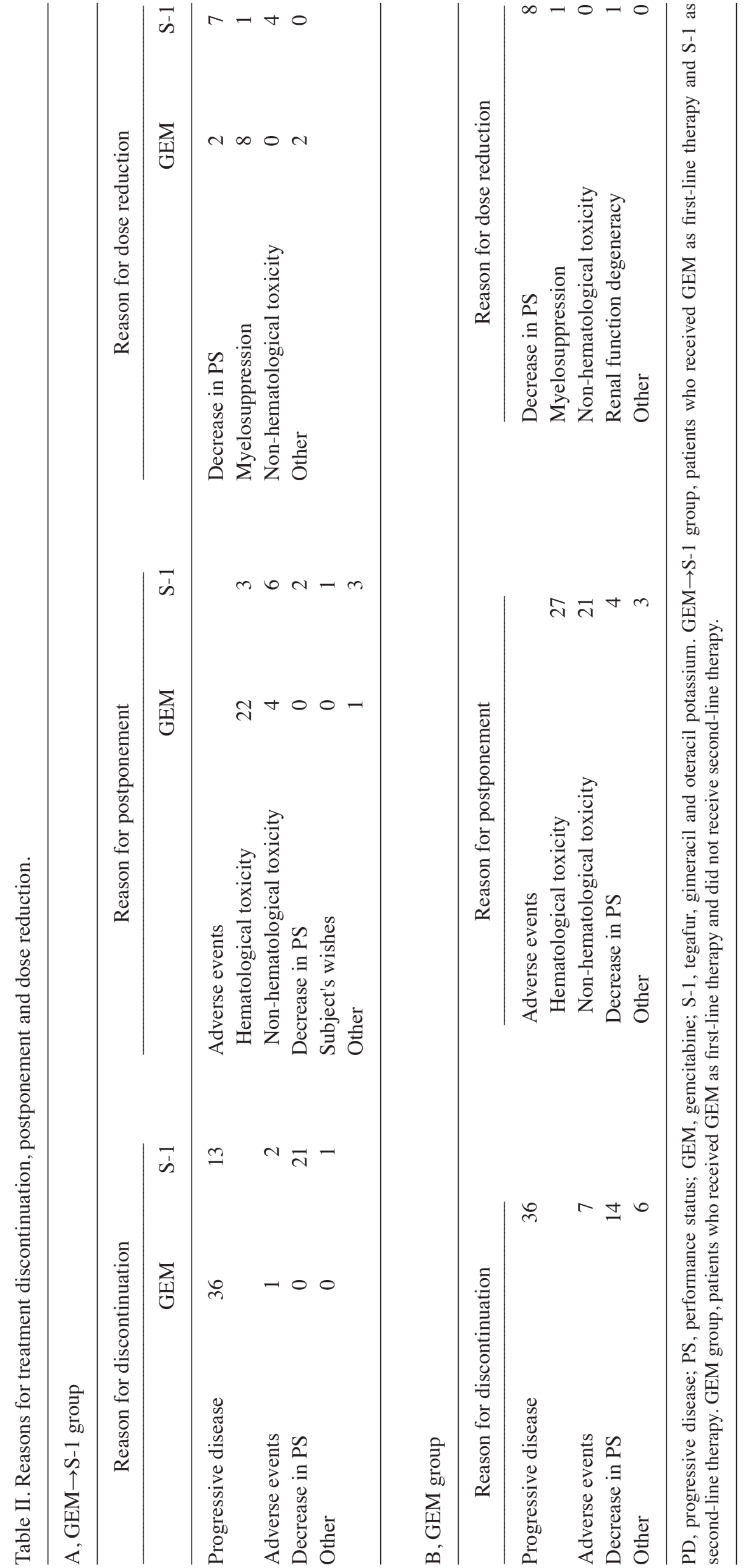


Table III. Adverse events following second-line therapy with S-1.

\begin{tabular}{|c|c|c|c|c|c|c|}
\hline \multirow[b]{2}{*}{ Adverse events } & \multicolumn{4}{|c|}{ Grade } & \multirow[b]{2}{*}{ All grades $(\%)$} & \multirow[b]{2}{*}{ Grade $\geq 3(\%)$} \\
\hline & 1 & 2 & 3 & 4 & & \\
\hline Oligochromemia & 1 & 9 & 4 & 0 & $14(37.8)$ & $4(10.8)$ \\
\hline Leukopenia & 4 & 2 & 1 & 0 & $7(18.9)$ & $1(2.7)$ \\
\hline Neutropenia & 2 & 2 & 2 & 0 & $6(16.2)$ & $2(5.4)$ \\
\hline AST/ALT increase & 3 & 0 & 1 & 0 & $4(10.8)$ & $1(2.7)$ \\
\hline Blood bilirubin increase & 2 & 1 & 0 & 0 & $3(8.1)$ & $0(0.0)$ \\
\hline Creatinine increase & 1 & 2 & 0 & 0 & $3(8.1)$ & $0(0.0)$ \\
\hline Anorexia & 4 & 3 & 0 & 0 & $7(18.9)$ & $0(0.0)$ \\
\hline Diarrhoea & 3 & 4 & 0 & 0 & $7(18.9)$ & $0(0.0)$ \\
\hline Malaise & 4 & 2 & 0 & 0 & $6(16.2)$ & $0(0.0)$ \\
\hline Stomatitis & 4 & 2 & 0 & - & $6(16.2)$ & $0(0.0)$ \\
\hline Nausea & 3 & 1 & 1 & 0 & $5(13.5)$ & $1(2.7)$ \\
\hline Watering eyes & 5 & 0 & 0 & - & $5(13.5)$ & $0(0.0)$ \\
\hline Skin hyperpigmentation & 4 & 0 & - & - & $4(10.8)$ & $0(0.0)$ \\
\hline Rash & 3 & 0 & 0 & 0 & $3(8.1)$ & $0(0.0)$ \\
\hline Hand-foot syndrome & 2 & 0 & 1 & 0 & $3(8.1)$ & $1(2.7)$ \\
\hline Oedema & 1 & 2 & 0 & 0 & $3(8.1)$ & $0(0.0)$ \\
\hline
\end{tabular}

S-1, tegafur, gimeracil and oteracil potassium; AST, aspartate aminotransferase; AST, alanine aminotransferase.

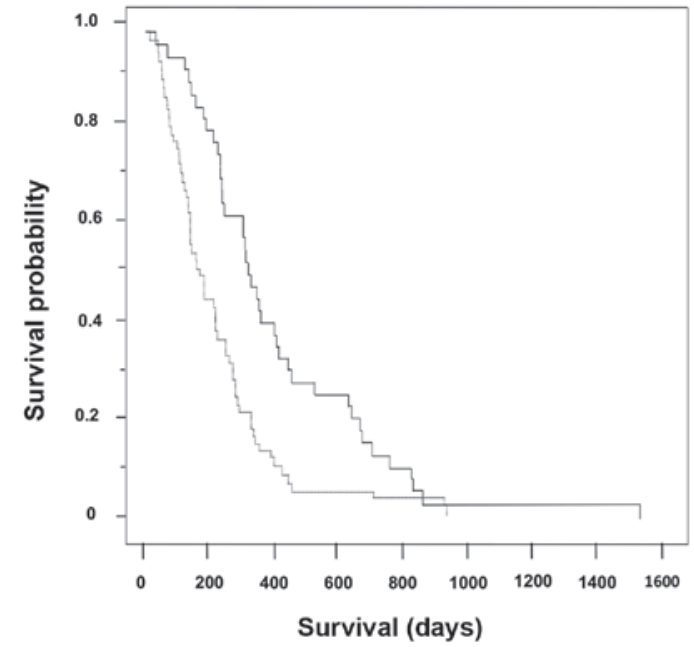

Figure 1. Kaplan-Meier survival curves of overall survival in the gemcitabine $($ GEM $) \rightarrow$ S-1 and GEM groups. Solid line, GEM $\rightarrow$ S-1group. Median survival time (MST), 323 (64-1514) days. Dotted line, GEM group. MST, 172 (33-918) days.

pharmacodynamics rather than pharmacokinetics determines the effect of GEM on survival.

Therefore, we recommend avoiding dosage reduction when the neutrophil count is high. These findings may aid future evaluation of dose escalation in patients without neutropenia to prolong survival. Prospective trials are required to assess whether dosing adjustments based on neutropenia may improve chemotherapeutic efficacy.

Regarding the safety of second-line therapy with S-1, grade $\geq 3$ haematological toxicities were observed, but non-haematological toxicities were rarely recorded. The main

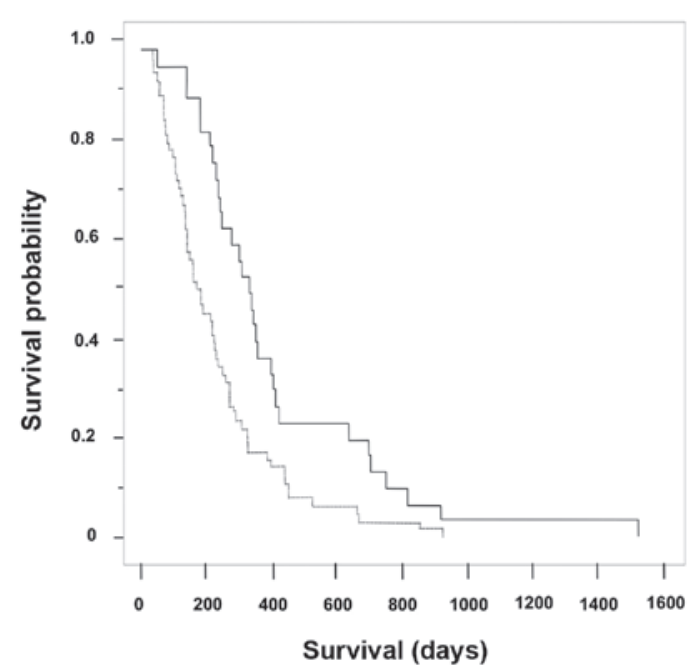

Figure 2. Kaplan-Meier survival curves showing the highest grade of neutropenia following first-line therapy with gemcitabine (GEM). Solid line, patients who had severe neutropenia (grade $\geq 3$ ) during treatment with GEM. Median survival time (MST), 330 (47-1514) days. Dotted line, patients who had mild neutropenia (grade $\leq 2$ ) during treatment with GEM. MST, 178 (33-918) days.

AEs observed following second-line therapy with S-1 included haematological toxicities, such as oligochromemia (37.8\%), leukopenia (18.9\%) and neutropenia (16.2\%), and non-haematological toxicities, such as anorexia (18.9\%), diarrhoea (18.9\%), malaise (16.2\%) and stomatitis (16.2\%), which were also reported by Todaka et al (18). In addition, of the 37 patients who received S-1 therapy, treatment was discontinued in 2 cases due to non-haematological toxicities (diarrhoea and anorexia). By contrast, in 6 cases with 
non-haematological toxicities, such as diarrhoea, stomatitis, hand-foot syndrome, constipation, anorexia and vomiting, S-1 therapy was safely continued by postponing treatment. However, during second-line therapy with S-1, the frequency of grade $\geq 2$ fatigue and gastrointestinal toxicity was $27.0 \%$ (10/37 cases) in patients with Alb levels $<3.5 \mathrm{~g} / \mathrm{dl}$. This result is similar to that of previous studies $(15,16)$ and should be considered when treating patients with $\mathrm{S}-1$, as it may affect the treatment course. Similarly, fatigue and gastrointestinal toxicity in APC chemotherapy patients with Alb levels $<3.5 \mathrm{~g} / \mathrm{d}$ l must be carefully considered when planning the chemotherapy protocol.

In conclusion, neutropenia occurring when GEM is administered as first-line treatment to APC patients is strongly associated with a better prognosis. S-1 therapy as second-line treatment has been associated with a low incidence of severe AEs and the patients were able to successfully continue treatment.

\section{References}

1. Matsuda T, Ajiki W, Marugame T, Ioka A, Tsukuma H and Sobue T; Research Group of Population-Based Cancer Registries of Japan: Population-based survival of cancer patients diagnosed between 1993 and 1999 in Japan: A chronological and international comparative study. Jpn J Clin Oncol 41: 40-51, 2011.

2. Burris HA III, Moore MJ, Andersen J, Green MR, Rothenberg ML, Modiano MR, Cripps MC, Portenoy RK, Storniolo AM, Tarassoff $\mathrm{P}$, et al: Improvements in survival and clinical benefit with gemcitabine as first-line therapy for patients with advanced pancreas cancer: A randomized trial. J Clin Oncol 15: 2403-2413, 1997.

3. Ueno H, Ioka T, Ikeda M, Ohkawa S, Yanagimoto H, Boku N, Fukutomi A, Sugimori K, Baba H, Yamao K, et al: Randomized phase III study of gemcitabine plus S-1, S-1 alone, or gemcitabine alone in patients with locally advanced and metastatic pancreatic cancer in Japan and Taiwan: GEST study. J Clin Oncol 31: 1640-1648, 2013.

4. Hatori M, Tsuji D, Taku K, Daimon T, Kamezato M, Ikeda M, Makuta R, Hayashi H, Inoue K and Itoh K: Prognostic factors in patients with unresectable pancreatic cancer treated with gemcitabine: a retrospective analysis. Jpn J Pharm Health Care Sci 40: 734-741, 2014.

5. Teramukai S, Kitano T, Kishida Y, Kawahara M, Kubota K, Komuta K, Minato K, Mio T, Fujita Y, Yonei T, et al: Pretreatment neutrophil count as an independent prognostic factor in advanced non-small-cell lung cancer: An analysis of Japan Multinational Trial Organisation LC00-03. Eur J Cancer 45: 1950-1958, 2009.

6. An X, Ding PR, Li YH, Wang FH, Shi YX, Wang ZQ, He YJ, $\mathrm{Xu}$ RH and Jiang WQ: Elevated neutrophil to lymphocyte ratio predicts survival in advanced pancreatic cancer. Biomarkers 15: 516-522, 2010.
7. Stotz M, Gerger A, Eisner F, Szkandera J, Loibner H, Ress AL, Kornprat P, AlZoughbi W, Seggewiesn FS, Lackner C, et al: Increased neutrophil-lymphocyte ratio is a poor prognostic factor in patients with primary operable and inoperable pancreatic cancer. Br J Cancer 109: 416-421, 2013.

8. Shitara K, Matsuo K, Takahari D, Yokota T, Shibata T, Ura T, Ito S, Sawaki A, Tajika M, Kawai H and Muro K: Nuetropenia as a prognostic factor in advanced gastric cancer patients undergoing second-line chemotherapy with weekly paclitaxel. Ann Oncol 21: 2403-2409, 2010.

9. Shitara K, Matsuo K, Oze I, Mizota A, Kondo C, Nomura M, Yokota T, Takahari D, Ura T and Muro K: Meta-analysis of neutropenia or leukopenia as a prognostic factor in patients with malignant disease undergoing chemotherapy. Cancer Chemother Pharmacol 68: 301-307, 2011.

10. Shitara K, Matsuo K, Takahari D, Yokota T, Inaba Y, Yamaura H, Sato Y, Najima M, Ura T and Muro K: Neutropaenia as prognostic factor in metastatic colorectal cancer patients undergoing chemotherapy with first-line FOLFOX. Eur J Cancer 45: 1757-1763, 2009 .

11. Kawashima H, Itoh A, Ohno E, Nakamura M, Miyahara R, Ohmiya N, Hara K, Kanamori A, Itoh T, Taki T, et al: Prospective multicenter study to investigate the introduction rate of second-line S-1 in gemcitabine-refractory unresectable pancreatic cancer. Cancer Chemother Pharmacol 68: 677-683, 2011.

12. Nakai Y1, Isayama H, Sasaki T, Sasahira N, Kogure H, Hirano K, Tsujino T, Ijichi H, Tateishi K, Tada M, et al: Impact of S-1 in patients with gemcitabine-refractory pancreatic cancer in Japan. Jpn J Clin Oncol 40: 774-780, 2010.

13. Nakai Y1, Isayama H, Sasaki T, Sasahira N, Ito Y, Kogure H, Togawa O, Matsubara S, Arizumi T, Yagioka H, et al: Impact of S-1 on the survival of patients with advanced pancreatic cancer. Pancreas 39: 989-993, 2010

14. Takasawa O, Fujita N, Noda Y, Kobayashi G, Ito K, Obana T, Horaguchi J, Koshita S, Kanno Y, Suzuki T, et al: Second-line treatment with S-1 in advanced pancreatic cancer. Gastroenterology 48: 207-213, 2009.

15. Kimura M, Morihata K, Ito D, Iwai M, Okada K, Usami E, Nakao T, Yoshimura T and Yasuda T: Continuous administration and safety of S-1 in adjuvant chemotherapy for gastric cancer. Cancer \& chemotherapy 37: 829-834, 2010 (In Japanese).

16. Kimura M, Usami E, Yoshimura T, Yasuda T, Kaneoka Y, Teramachi H, Sugiyama T and Tsuchiya T: Pharmaceutical care for patients undergoing S-1 plus cisplatin therapy for unresectable recurrent gastric cancer. J Pharm Pract 26: 409-414, 2013.

17. Fridlender ZG and Albelda SM: Tumor-associated neutrophils: Friend or foe? Carcinogenesis 33: 949-955, 2012

18. Todaka A, Fukutomi A, Boku N, Onozawa Y, Hironaka S, Yasui H, Yamazaki K, Taku K, Machida N, Sakamoto T and Tomita H: S-1 monotherapy as second-line treatment for advanced pancreatic cancer after gemcitabine failure. Jpn J Clin Oncol 40: 567-572, 2010. 